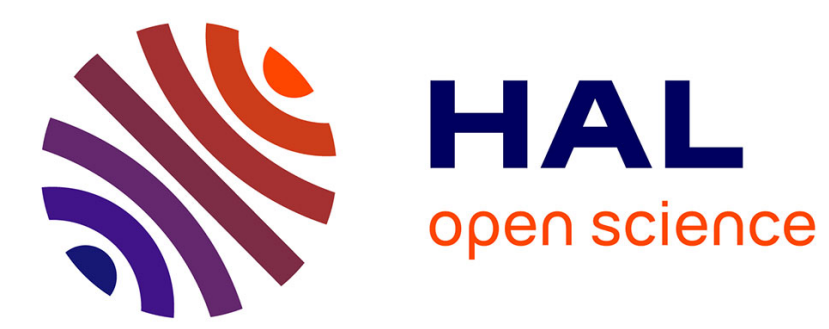

\title{
Large Strain Range Dynamic Testing at High and Medium Strain Rates, Using a Common Scale SHPB H. Zhao, G. Gary
}

\section{To cite this version:}

H. Zhao, G. Gary. Large Strain Range Dynamic Testing at High and Medium Strain Rates, Using a Common Scale SHPB. Journal de Physique IV Proceedings, 1997, 07 (C3), pp.C3-341-C3-346. 10.1051/jp4:1997360 . jpa-00255517

\section{HAL Id: jpa-00255517 https://hal.science/jpa-00255517}

Submitted on 1 Jan 1997

HAL is a multi-disciplinary open access archive for the deposit and dissemination of scientific research documents, whether they are published or not. The documents may come from teaching and research institutions in France or abroad, or from public or private research centers.
L'archive ouverte pluridisciplinaire HAL, est destinée au dépôt et à la diffusion de documents scientifiques de niveau recherche, publiés ou non, émanant des établissements d'enseignement et de recherche français ou étrangers, des laboratoires publics ou privés. 


\title{
Large Strain Range Dynamic Testing at High and Medium Strain Rates, Using a Common Scale SHPB
}

\author{
H. Zhao and G. Gary
}

Laboratoire de Mécanique des Solides, École Polytechnique, 91128 Palaiseau, France

\begin{abstract}
Résumé : La durée de la mesure d'un système à bartes de Hopkinson est limitée par la longueur des barres à cause de la superposition algébrique des on les se propageant dans des sens opposés. Il en résulte que la déformation maximale mesurable dans un essai de comportement est limitée. Nous présentons une version améliorée de la méthode des deux jauges qui tient compte de la dispersion des ondes et conduit à une mesure quasi illimitée dans la durée (une centaine de fois la durée conventionnelle). Cette méthode est appliquée aux essais de matériaux de faible résistance tels que les mousses (polymériques ou métalliques) pour obtenir les grandes déformations désirées ( $80 \%)$ à faible vitesse de déformation (10/s). Elle est également uilisée pour réaliser des essais de compression dynamique de surctures avec déplacements importants (crash des tubes métalliques absorbeurs d'energie).
\end{abstract}

\begin{abstract}
The measuring duration of a SHPB (Split Hopkinson Pressure Bar) set-up is limited by the length of the bars because of the superimposition of the waves propagating in opposite directions, so that there exists a limitation of maximum measurable strains in material testing applications. This paper presents a new twogauges measurement method taking account of the correction of wave dispersion etfects. as it is indeed indispensable for long time measurements. Using bars of common dimensions. it allows for a quasi-unlimited measuring duration (one hundred times longer than the classical one. It is applied to test soft materials like foam (metallic or polymeric) at low strain-rates $(10 / \mathrm{s})$, in the complete range of their response (nominal strains up to $80 \%$ ). It is also successfully used to perform large displacement tests such as crushing of metallic tubes.
\end{abstract}

\section{INTRODUCTION}

The SHPB (Split Hopkinson Pressure Bar) is nowadays a standard experimental technique for performing tests under dynamic loading. Its success is mainly due to the accuracy of its measurements. The technique finds its origin in the works of Hopkinson [1] to mealsure a pressure-pulse profile using a long thin bar and it has been well established since the critical study made by Duvies [2]. The practical configuration with two long bars and a short specimen between them, widely used today, was introduced by Kolsky [3]. This technique, initially developed for compression tests, has been applied to tensile loading [4] and to torsional loading [5]. To improve its precision, wave dispersion effects $[2,6,7,8]$, threedimensional effects $[9,10,11]$ and transient effects $[12,13,14,15]$ in the specimen have been studied in the past decades.

The measuring technique using bars relies on the knowledge of the two clementary waves propagating in opposite directions. Once they are known, they can be time-shifted to the desired cross-section (bar-specimen interfaces for example) to calculate forces and velocities. The SHPB technique uses long bars and a short loading pulse so that there exists a cross-section where the total incident pulse and the first part of reflected waves (of the same duration) can be recorded separately. There exists then a maximum observation duration depending on the length of the bars. This measuring duration $\Delta T$ of a classical SHPB set-up is limited to $\Delta T \leq L / C, C$ being wave speed and $L$ the length of the bar. Consequently, the total relative displacement $\Delta l$ between the two bar-specimen interfaces is limited for a given loading speed $\mathrm{V}(\Delta l \leq \mathrm{V} \Delta T)$. For material behaviour testing at a given average strain rate, the maximum measurable strain is also limited $\left(\varepsilon_{\max } \leq \dot{\varepsilon} \Delta T\right)$. For instance. the meatsurable duration does not exceed 400us for a SHPB set-up made of $2 \mathrm{~m}$ long steel bars $(C \equiv 5000 \mathrm{~m} / \mathrm{s})$ and the measurable maximum strain is limited to $20 \%$ for a test performed at an average strain rate of $500 \mathrm{~s}^{-1}$ (and only $1 \%$ for $25 \mathrm{~s}^{-1}$ ). 
The SHPB technique then fails in two testing situations. One case is when the desired maximum stati (or total relative displacement) is very important. For example, in the study of the dynamic behaviour polymeric or metallic foam, the desired maximum strain is often up to $80 \%$ to investigatte thi densification phenomenon of those materials. A large displacement during the test is also required for thi study of the dynamic buckling of thin metallic tubes. In fact, specimens are effectively submitted to the desired strains (because of their low resistance) during the total time remaining after the observilisin window, but measurements are no longer possible after this time in a standard SHPB. Another calse is when the desired strain rate is in the medium range. The technique can not be applied because th: maximum measurable strain becomes too small.

To increase the measuring duration of SHPB, some earlier workers have analysed the muluple reflections in bars. Campbell and Duby [16] have reported a method on the basis of one dimensiomil elastic wave theory. Lundberg and Henchoz [17] have also proposed a simple explicit formula (within? one-dimensional wave propagation assumption) to separate the two elementary waves and to measure the particle velocity after the observation window, using two signals recorded at two different cross-sections in a bar. Recently, this method has found a new application [18] in the prediction of the wave propagattin: in a bar with a non-uniform impedance (due to a temperature gradient, for instance) and successfully uxd in high temperature SHPB testing [19,20]. However, as indicated by those atuthors [16,17], such a metlind is valid only if the wave dispersion effect can be neglected. This situation is found when the bar is thir and the measuring duration is short (the duration in those works is about $1 \mathrm{~ms}$ for a bar of 10 mInt diameter).

This paper presents a new measuring method on the basis of a sinilar analysis but without abu: limitations. An iterative formula allowing for the calculation of the two virtually separated elementary waves for the total desired testing duration is presented, using two signals recorded at two differcm cross-sections in each bar. It takes into account the wave dispersion effects which are very important lir the accuracy of long time measurements.

\section{SEPARATION OF WAVES PROPAGATING IN OPPOSITE DIRECTIONS IN A BAR}

\subsection{Two strain gauges wave separation method}

The classical measuring technique using bars is based on the mechanics of elastic wave propagation i: bars and on the superposition principle. Considering the two elementary waves in a bar, the wille propagating in the positive direction (arbitrarily defined) is named the "ascending" wave and the other one the "descending" wave (to avoid the confusion with the classical SHPB, the terms "incident wilv" and "reflected wave" are not used here). The strain $\varepsilon(t)$ at each section is the sum of the contribution of the elementary "ascending" wave $\varepsilon_{\text {asc }}(t)$ and that of the elementary "descending" wave $\varepsilon_{\text {des }}(t)$.

$$
\varepsilon(t)=\varepsilon_{\text {taxc }}(t)+\varepsilon_{d e s}(t) \text {, where } C_{0} \text { is the wave speed. }
$$

At the section where the strain is measured, a prior knowledge of the contribution of one elementart? wave will allow for the calculation of the other one. Two strain gages are cemented at distinct points 1 and $B$ of the bar, as shown in Figure 1 . The strain histories at those two points $\varepsilon_{i t}(t)$ and $\varepsilon_{B}(t)$ all recorded. Since the bar is loaded at one end (by the projectile for the input bar and by the specimen for the output bar) while the other end remains at rest, the recorded signal at the first measuring crosssection $\mathrm{A}$ is due to a single elementary wave until the reflection at the other end comes back. Thi "ascending" wave at $A \varepsilon_{\text {arc. }}(t)$ (because of positive direction of z-axis) is then equal to the measurement at point $\mathrm{A}$, for the period $t<\mathrm{R}_{\mathrm{A}}$. It can be said for the same reason that the "descending" wave at point $\mathrm{B}$ $\varepsilon_{i k ; i s}(t)$ is equal to zero for the period $t<R_{B}$ :

$$
\varepsilon_{a t+A}(t)=\varepsilon_{A}(t) \quad \text { if } t<R_{A}, \quad \varepsilon_{t k+B}(t)=0 \quad \text { if } t<R_{b} .
$$


In order to exhibit "ascending" and "descending" waves from measurements at A and B which become afterwards sum of both elementary waves, an iterative process is built. Strain measurements $\varepsilon_{A}(t)$ and $\varepsilon_{B}(t)$ are divided into small pieces $\varepsilon_{.4}^{i}(t)$ and $\varepsilon_{B}^{i}(t)$ of a constant time length $\Delta \mathrm{t}$ as shown in Figure 1. This interval $\Delta \mathrm{t}$ is twice the time needed by the waves to travel between the two gages. In other

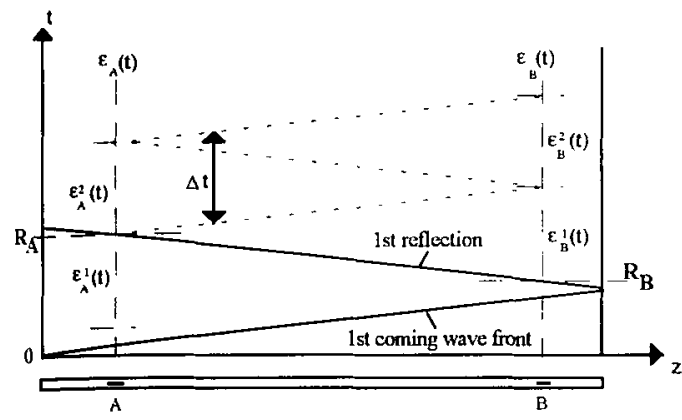

Figure 1. Scheme for the separation of the waves words, $\varepsilon_{A}^{l}(t), \varepsilon_{. i}^{2}(t) \ldots \varepsilon_{. i}^{\prime}(t)$ and $\varepsilon_{B}^{l}(t), \varepsilon_{B}^{2}(t) \ldots \varepsilon_{B}^{\prime}(t)$ are obtained through the characteristic function of the intervals $[(i-1) \Delta \mathrm{t}, i \Delta \mathrm{t}](\mathrm{i}=1,2,3 \ldots)$ :

$$
\begin{aligned}
& \varepsilon_{A}^{i}(t)= \begin{cases}\varepsilon_{A}(t) & R_{A}+(i-2) \Delta t \leq t \leq R_{A}+(i-1) \Delta t \\
0 & \text { otherwise }\end{cases} \\
& \varepsilon_{B}^{i}(t)= \begin{cases}\varepsilon_{B}(t) & R_{B}+(i-1) \Delta t \leq t \leq R_{B}+i \Delta t \\
0 & \text { otherwise }\end{cases}
\end{aligned}
$$

This operation can be applied to both virtual elementary "ascending" and "descending" waves at points $\mathrm{A}$ and $\mathrm{B}$ to obtain $\varepsilon_{a s c A}^{i}(t), \varepsilon_{a s c B}^{i}(t), \varepsilon_{d e s A}^{i}(t)$ and $\varepsilon_{d e s B}^{i}(t)$. According to Eqn. (2), the "ascending" wave at $\mathrm{A}$ for $\mathrm{i}=\mathrm{l}\left(R_{A}-\Delta t \leq t \leq R_{A}\right), \varepsilon_{\text {ascA }}^{\prime}(t)$ is known. Knowing how the waves propagate, the "ascending" wave at point $\mathrm{B}$ is found by applying the shifting function to the "ascending" wave at point A.

$$
\varepsilon_{\text {ascB }}^{1}(t)=f_{\text {shipl }}\left(\varepsilon_{\text {ascA }}^{1}(t)\right) \text {. }
$$

Using Eqn. (I) at point $\mathrm{B}$ for $\mathrm{i}=1\left(R_{B} \leq t \leq R_{B}+\Delta t\right)$, the corresponding part of the "descending" wave at point $B$ is

$$
\varepsilon_{d e s B}^{l}(t)=\varepsilon_{B}^{1}(t)-\varepsilon_{a s c B}^{1}(t)
$$

The same process is performed to calculate the "ascending" wave at point A for the next interval from the knowledge of the "descending" wave given by (5). Following this way, an iterative formula is constructed and both "ascending" and "descending" waves can be calculated for all the time intervals.

\subsection{Introduction of the wave dispersion correction in the iterative process.}

Even for a classical SHPB set-up (where measured waves are at most shifted one length of the bar), the one-dimensional wave propagation theory has been shown not to be accurate enough $[2,7,8,21,22]$. If the wave dispersion effects are not taken into account, the accuracy of the two-strain measurement method becomes rapidly insufficient with the increase of the propagation distance, as indicated by Lundberg and Henchoz [17]. Consequently, a more accurate propagation theory must be used, as it has already been introduced in the SHPB to improve the accuracy of the shifting process, taking account of so called wave dispersion effects.

The analytical solution of the propagation of longitudinal waves in an infinite elastic bar has been used in different cases $[2,6,7,8,21,22,23]$. This solution has been extended to bars made of a linear viscoelastic material [24]. Such bars (made of Nylon or PMMA) are needed to achieve an improved impedance ratio when soft material such as polymeric foam is studied. Even already emphasised by Davies [2], the infinite bar theory is not theoretically exact but it has been verified in the usual SHPB set-up that it gives very good results.

It is then natural to use this wave propagation theory [25]. Such an approach relies on the assumption that waves in the bar are harmonic so that all the associated mechanical variables can be described by 
their harmonic components. The dispersion relation $\xi=\xi(\omega)$ between the wave number $\xi$ and $t_{i}$ frequency $\omega$, describing the propagation of each frequency component, is defined through the so-cilled frequency equation derived from the solution of the three-dimensional wave propagation in an infinie bar. The wave shifting between $A$ and $B$ (separated by the distance $\Delta z=z_{B}-z_{1}$ ) consists in multiplying frequency components by a term depending on the dispersive relation

$$
\varepsilon_{B}^{*}(\omega)=\varepsilon_{A}^{*}(\omega) e^{i \xi(\omega) \Delta z} .
$$

For the time separating scheme presented in $\$ 2.1$, the shifting function used in Eqn. (4) is obtained with the wave propagation theory (Eqn. 6) and evaluated numerically using the Fast Fourier Translom (FFT) :

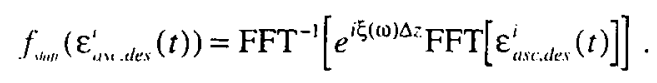

It is noted that the time separation scheme becomes theoretically not exact because of th: incompatibility between the temporal cuts and the infinite stationary wave propagation theory. Fir example, the interval of duration $\Delta t$ has been previously defined as " twice the time needed by the watc: for a travel between two gages $»$ but a common value with respect to frequency components of $\Delta t$ cannot be defined because the wave velocity now depends on the frequency. However, this temporal separating scheme produces cumulative errors so that the quality of the results only decreases with the distance virtually covered by the waves. The result within a certain distance is quite accurate [26].

\section{APPLICATIONS TO SHPB TECHNIQUE TO IMPROVE MEASURING DURATION}

\subsection{Behaviour of polymers and polymeric foam under dynamic loading.}

Recently, the knowledge of the behaviour of low impedance materials (like foams) under carti situations has been needed in the automotive industry and has appeared as an important goal. Ont particular feature of foam testing is the need for a large maximum strain (up to 80\%) in order to study the densification part of the response, associated with a significant increase of the stress. In a conventional SHPB test at quite high strain rates of about $300 / \mathrm{s}$, it is not possible to measure such strains, even if they are easily reached after the measuring phase because of the very low resistance it foam. The difficulty lying in a limited measuring duration is solved with the wave separation methol presented here.

A test on polymeric foam (a specimen of $40 \mathrm{~mm}$ of length and $40 \mathrm{~mm}$ of diameter) using a common Nylon SHPB with two $3 \mathrm{~m}$ bars of a diameter of $40 \mathrm{~mm}$ is taken for an example. Two strain gilles (instead of one) are cemented on the input and output bars. The four strain histories are recorded illd shown in Figure 2a (gages 1,2 are on the input bar, gages 3,4 on the output bar). One can recognise from the signal of gage 2 that a common quasi-rectangular incident wave is launched by the projectili and that this pulse makes periodic round trips in the input bar.

The input and output velocities calculated from those signals are illustrated in Figure $2 b$. The measurable duration is about $12 \mathrm{~ms}$, which is 10 times the initial SHPB measuring duration (about l.? $\mathrm{ms}$ ) for this set-up. In terms of measurement duration, it is as if two bars of $30 \mathrm{~m}$ long had been used. Thi deduced average stress-strain relation is shown in Figure 3. The material behaviour is measured up 10 it strain 3 times larger than for a conventional SHPB. Since there is a periodic loading due to the round trip of the incident wave in the input bar, 4 loading-unloading cycles are observed. Unloading and reloadin: curves do not follow exactly the same path, which is in agreement with known material characteristic and gives confidence in the accuracy of the measurement. The capability to obtain dynamic loadin:unloading cycles could be interesting for some other viscoelastic or materials which accumulate damagc: If the unloading has to be avoided, one just has to use a different and better adapted loading device suklt 
as the "slow bar" [26], which allow tests at medium strain rates $(5 / \mathrm{s}$ to $50 / \mathrm{s})$. A comparison of results at $250 / \mathrm{s}$ and at $30 / \mathrm{s}$ is given in the figure 4 , showing the strong rate sensitivity of the tested foam.
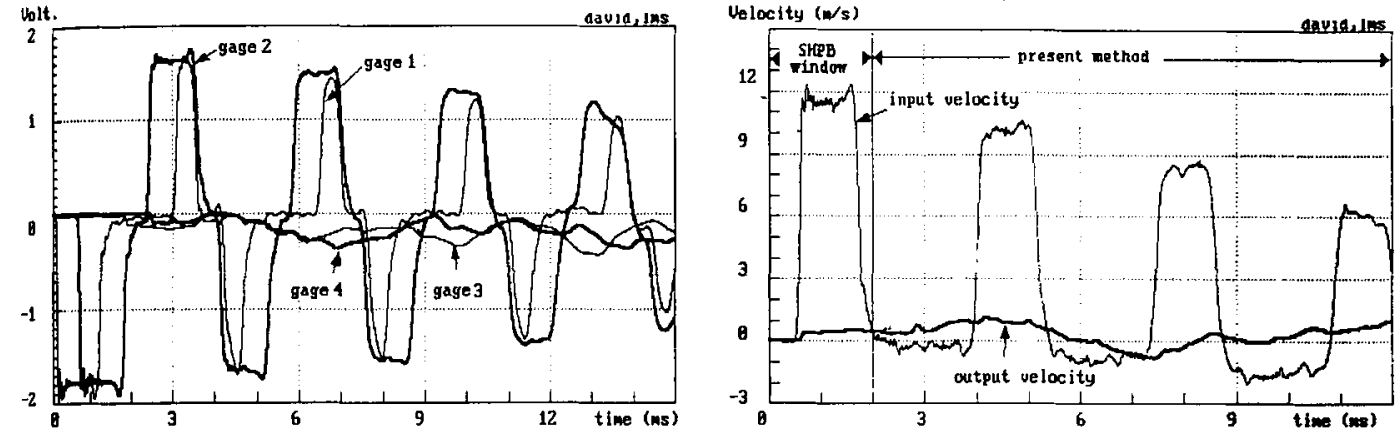

Fig.2a Recordings of gages cemented on input and output bars.

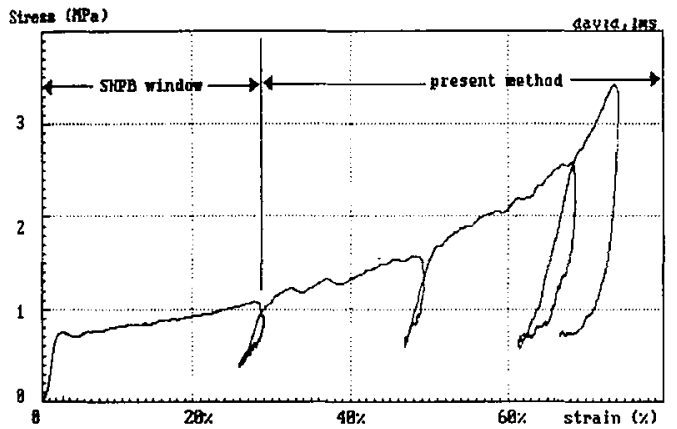

Fig.3 Average stress-strain relation of the foam
Fig.2b Calculated input and output velocities

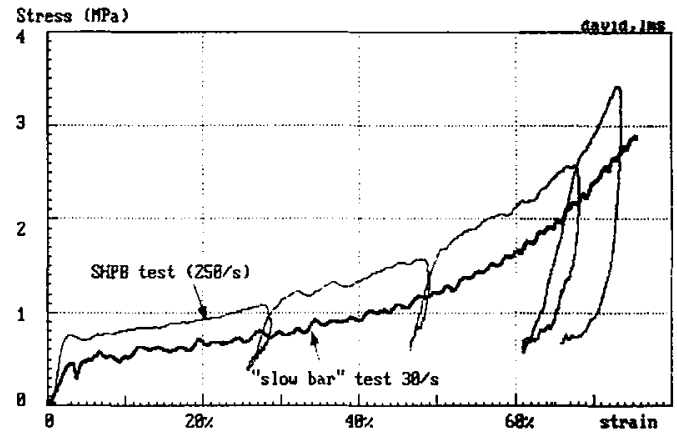

Fig.4 Strain rate effects on foam at medium strain rates

\subsection{Buckling of metallic tubes under dynamic loading}

Metallic thin tubes are widely used in the automotive industry, essentially as energy absorbers. Crush tests of tubes are usually made with a falling mass testing device. The deceleration of that mass is measured by an accelerometer. The associated force and displacement are then deduced, but the measuring precision is not always very good because of the vibrations of the weight and its suspension

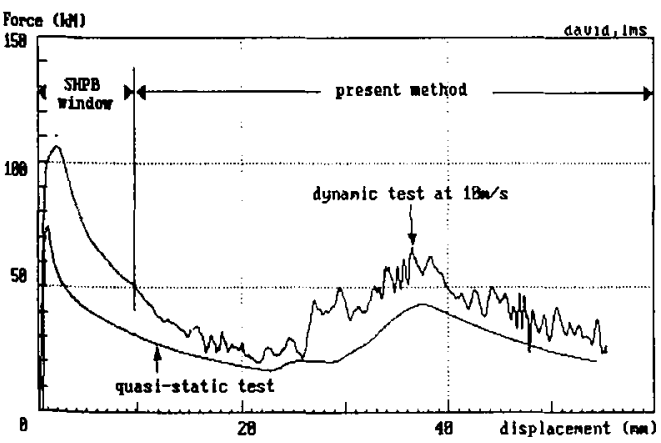

Figure 5. Quasi-static and SHPB tests on an aluminium square thin tube

system. It is thus of interest to use a SHPB system, known for its measuring precision, to test these kinds of structures. However, this application requires a SHPB bar with a large diameter to cover the tube section. In our laboratory, a large diameter $(80 \mathrm{~mm})$ SHPB system is used for this application. In its classical version, this set-up with a $6 \mathrm{~m}$ long input bar made of aluminium provides an observable applied displacement of about $10 \mathrm{~mm}$ for an impact at $10 \mathrm{~m} / \mathrm{s}$. Using the new method presented in section 2, tests of mini-structures can be performed with a large applied displacement.

Figure 5 shows testing results on an aluminium $50 \times 50 \mathrm{~mm}$ square tube (thickness of $2 \mathrm{~mm}$ ). A projectile of $3 \mathrm{~m}$ made of steel is used to produce a more important loading duration without unloading (the impacting side of the input bar remains loaded for a long time because the impedance of the projectile is greater than that of the input bar). The presented 
method provides an observed crushing displacement of $60 \mathrm{~mm}, 6$ times greater than the displacement observed with the usual SHPB window. Oscillations after the limit of the classical SHPB measurement are observed in the final force-displacement relation (the force-displacement relation shown in Figurc + is a raw result without any smoothing). Nevertheless, the precision in our case is much better than lital obtained with a falling weight set-up.

\section{CONCLUSIONS}

A new two-gauges method allowing for an almost unlimited measuring duration with common scile bars is presented in this paper. This method is based on an iterative shifting process in which the wate dispersion effect is taken into account. The application to common SHPB systems permits a significinl increase (up to 100 times) in the measurement duration compared to a classical SHPB. This technique is illustrated with the testing of soft polyurethane foam at medium strain rates and the crushing of mini. structures with significant displacements.

\section{REFERENCES}

[1] Hopkinson, B., Phil. Trans. Roy. Soc., A213 (1914), 437-452.

[2] Davies, R.M., Phil. Trans. Roy. Soc., A240 (1948), 375-457.

[3] Kolsky, H., Proc. Phys. Soc., B62 (1949), 676-700.

[4] Harding, J. Wood, E.D. and Campbell, J.D. J. Mech. Engng. Sci., 2 (1960), 88-96.

[5] Duffy, J. Campbell, J.D. and Hawley, R.H., J. Appl. Mech. 38 (1971), 83-91

[6] Yew, E.H. and Chen, C.S. J. Appl. Mech., 45 (1978), 940-942.

[7] Follansbee, P.S. and Franz, C., J. Engng. Mater. Tech., 105 (1983), 61-66.

[8] Gorham, D.A. J. Phys. E: Sci. Instrum. 16(1983), 477-479.

[9] Davies, E.D.H. and Hunter, S.C., J. Mech. Phys. Solids, 11 (1963), 155-179.

[10] Bertholf, L.D. and Karnes, J., J. Mech. Phys. Solids, 23 (1975), 1-19.

[11] Malinowski, J.Z. and Klepaczko, J.R., Int. J. Mech. Sci., 28 (1986), 381-391.

[12] Lindholm U.S., J. Mech. Phys. Solids, 12 (1964), 317-335.

[13] Conn, A.F., J. Mech. Phys. Solids, 13(1965) , 311-327.

[14] Bell J.F., J. Mech. Phys. Solids, 14 (1966), 309-327.

[15] Jahsman, W.E. J. Appl. Mech. (1971), 77-82.

[16] Campbell, J.D. and Duby, J., Proc. R. Soc. Lond., A 236 (1956), 24-40.

[17] Lundberg, B. and Henchoz, A. Exper. Mech. 17 (1977), 213-218.

[18] Lundberg, B., Carlsson J. and Sundin K. G., 137 (1990), 483-493.

[19] Bacon, C. Färm, J. and Lataillade, J.L. Exper. Mech., 34(1994), 217-223.

[20] Lataillade, J.L., Bacon, C., Collombet, F. and Delaet, M. Wave Propagation and Emerging Technologies. (Eds. W.K.Kinra, R.J. Clifton and G.C. Johnson). AMD-Vol. 188, (1994). 85-93.

[21] Gong, J.C., Malvern, L.E. and Jenkins, D.A., J. Engng. Mater. Tech., 112 (1990), 309-314.

[22] Lifshitz, J.M. and Leber, H., Int. J. Impact Engng. 15 (1994), 723-733.

[23] Zhao, H., Gary, G. Int. J. Solids \& Structures, 33(1996), 3363-3375.

[24] Zhao, H., Gary, G. J. Mech. Phys. Solids. 43 (1995), 1335-1348.

[25] Zhao, H., Gary, G. C.R.Acad. Sci. Paris, 319 (1994), série II, 987-992.

[26] Zhao, H., Gary, G. J. Mech.Phys. Solids. Accepted for the publication. 